\section{CONTINUOUS SPINAL ANESTHESIA - A NEWBORN TECHNIQUE FOR TIMES OF NEED?}

S Carneiro Moreira, I Neri ${ }^{\star}$, R Silva. Centro Hospitalar de Lisboa Ocidental, Lisbon, Portugal

10.1136/rapm-2021-ESRA.59

Background and Aims Described during World War II, continuous spinal anesthesia (CSA) enables continuous administration of intrathecal drugs, offering several anesthetic options versus single shot technique, although underutilised.

Here we present 4 cases of CSA performed for patients undergoing urgent upper abdominal and lower extremity orthopedic surgery during the COVID-19 pandemic. Our patients were ranked between 90-100 years old with ASA score IV, in which we highlight the presence of ischemic heart disease and chronic obstructive pulmonary disease.

Methods Invasive hemodynamic measurement was performed before CSA technique. Given the lack of microcatheter-overthe-needle kits in our center, we performed the technique using Perifix ${ }^{\circledR}$ Complete Set - B Braun, with full aseptic technique. The $18 \mathrm{G}$ Tuohy needle was introduced via a midline approach until CSF was encountered. The 20G catheter was then introduced $3 \mathrm{~cm}$ into the subarachnoid space. In all situations we opted for the administration of sufentanil $0.0025 \mathrm{mg}$ plus subsequent aliquots of hyperbaric bupivacaine $0.125 \%$, guided by sensory assessment of block level.

Results All surgeries were successfully concluded with minimal haemodynamic variation and avoidance of mechanical invasive ventilation. Patients were admitted in the PACU, where spinal catheters were removed, and continued their recovery at the speciality yard. No complications were observed, particularly post-dural puncture headache, neurologic or infectious events. Conclusions CSA is a useful anesthetic technique with a low failure rate. Its best role is likely to be in high-risk surgical patients, as it can provide excellent blockade conditions, with small doses of LA and little haemodynamic variation frequently seen with single shot techniques.

\section{PRILOCAINE AND CHLOROPROCAINE SPINAL ANAESTHESIA IN FRACTURED NECK OF FEMUR SURGERY}

1J Finnity*, 'M Gorecha, ${ }^{2} \mathrm{R}$ Erskine. 'George Eliot Hospital, Nuneaton, UK; ${ }^{2}$ University Hospitals of Derby and Burton, Derby, UK

10.1136/rapm-2021-ESRA.60

Background and Aims Spinal anaesthesia using bupivacaine with opioids is commonly used in fractured neck of femur surgery $(1,2)$. However this often results in a prolonged sympathetic block in the elderly causing significant hypotension which may require vasopressor support. Newer local anaesthetic agents such as prilocaine and chloroprocaine are already successfully used in elective orthopaedics and offer better cardiovascular stability with faster recovery $(3,4)$. We decided to assess the suitability of these newer agents in neck of femur surgery and review its outcomes.

Methods 200 patients having fractured neck of femur surgery were given spinal anaesthesia using either $2 \%$ prilocaine or 1\% 2-chloroprocaine for Hemiarthoplasty or Dynamic hip screw respectively. An additional femoral/lat. cutaneous nerve/ fascial iliaca block was performed at the start to allow patient positioning and provide an adjunct to increase the surgical operating time along with propofol sedation.

Results All patients had their surgery successfully without any conversions to general anaesthesia. We observed better cardiovascular stability intraoperatively and in recovery the blood pressure recording had returned to preoperative levels without any vasopressor support allowing faster recovery and discharge to the ward.

Conclusions We have shown that shorter acting spinal anesthetics can be used in neck of femur surgery successfully when combined with nerve blocks. But more importantly these agents offer significant advantages over bupivacaine by providing better heamodynamic stability and faster recovery whilst also avoiding hypotension once the patient has left the recovery area. More research is desperately needed to identify if these agents could improve mortality and if they should be adopted into international guidelines.

\section{VASOVAGAL SYNCOPE RELATED TO PAIN PROCEDURES IN A PAIN CLINIC AT A TERTIARY LEBANESE HOSPITAL BETWEEN 2016 AND 2019}

${ }^{1} \mathrm{~S}$ Chamandi* ${ }^{2} \mathrm{~S}$ Kamar, ${ }^{2} \mathrm{~S}$ Hallit. ${ }^{1}$ CHUNDS, Byblos, Lebanon; ${ }^{2}$ Holy Spirit University of Kaslik, Jounieh, Lebanon

\subsection{6/rapm-2021-ESRA.61}

Background and Aims A vasovagal reaction is a common cardiovascular complication that can occur during an interventional procedure. Our study focuses on evaluating the factors associated with vasovagal syncope (VVS) when having a pain procedure at the pain clinic as well as showing variation in vital signs associated with fainting across different periods of the procedure(before, during and after).

Methods A retrospective case control study was conducted in a university hospital in Lebanon (CHU-NDS) on adult Lebanese patients with data taken from the archives covering a 4 year period (2016-2019). It included 188 patients: 94 cases representing all the ones who had endured a VVS or

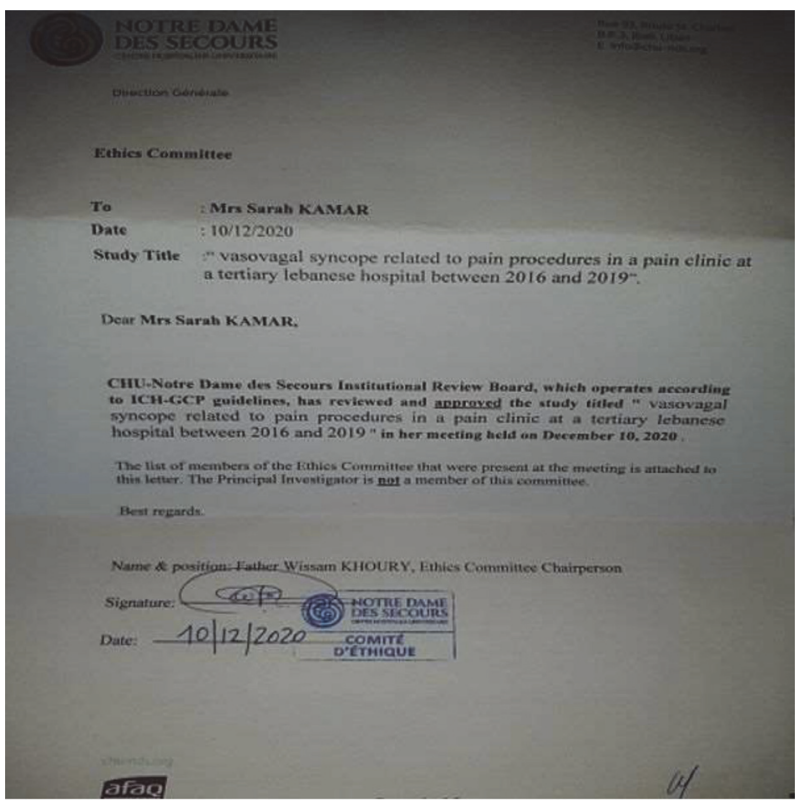

Abstract 61 Figure 1 